\title{
Refractive and Visual Outcomes of Different Intraocular Lenses with Femtosecond Laser Cataract Surgery: The Expectation of Independence from Spectacles
}

\author{
Joao Crispim* ${ }^{*}$ Ricardo Nose, Milton Yogi and Walton Nose \\ Department of Ophthalmology and Visual Sciences, Federal University of Sao Paulo-UNIFESP-Sao Paulo (SP), Brazil
}

\begin{abstract}
Purpose: To evaluate the visual performance of different types of intraocular lens (IOL) in eyes submitted to femtosecond laser (FSL) cataract surgery and to analyze whether this technology could result in spectacles independence.

Design: Retrospective comparative case series.

Methods: Consecutive eyes that were scheduled for FSL cataract surgery were included in this study. After one month postoperative, patients were divided into two groups: cases that required prescription for eyeglasses and cases that did not require prescription. In addition, the patients were analyzed according to the type of IOL that was implanted (monofocal, monofocal toric, multifocal and multifocal toric).

Results: Thirty-five cataract surgeries were analyzed. Twenty-three eyes had a monofocal IOL implanted, and 12 had a multifocal IOL implanted. After 1 month, 12 cases required prescription (Group 1), and 23 did not require prescription (Group 2). The proportion of eyes that did not require corrective lenses was significantly greater $(\mathrm{P}=0.02)$ in eyes that received multifocal IOL (91.67\%) compared with those that received monofocal IOL (47.83\%). Additionally, $100 \%$ of eyes that received multifocal toric IOL did not require eyeglasses at one month postoperatively.

Conclusion: The FSL surgical precision associated with modern IOL technology may be an important factor related to vision satisfaction after cataract surgery.
\end{abstract}

Keywords: Cataract surgery, femtosecond laser, intraocular lens, refractive surgery, spectacles independence.

\section{INTRODUCTION}

Femtosecond laser (FSL) has been used in cataract surgery to promote greater precision during crucial surgical stages: lens fragmentation, anterior capsulotomy and corneal incisions. It presents an opportunity for improvement in safety and efficacy, resulting in a more accurate and precise anterior capsulotomy, which affects IOL positioning and centering [1].

Although evidenced in only a limited number of studies, visual and refractive outcomes may gain many potential benefits through FSL that are at least as good as those of conventional phacoemulsification [2]. The benefit of a more stable intraocular lens (IOL) position, caused by a more precise capsulotomy, is a more predictable IOL power calculation [3]. Sutton et al. [1] reviewed key issues to consider in clinical practice when transitioning to FSLassisted cataract surgery. They mentioned a series of eyes with FSL-assisted cataract surgery and multifocal IOL implantation, with visual acuity comparable with manual phacoemulsification cohort, although with an improved level of unaided visual acuity.

*Address correspondence to this author at the Department of Ophthalmology and Visual Sciences, Federal University of Sao Paulo-UNIFESP-Sao Paulo (SP), R. Botucatu 821 Sao Paulo SP 04023-062 Brazil;

Tel: + 55115576 4000; Fax: + 55115576 4981;

E-mail: joaocrisp@gmail.com
In general, patients who are undergoing cataract surgery consider the opportunity to be free of glasses as being very important. With multifocal IOLs, the majority of patients do not wear glasses after cataract surgery, which is a potential source of savings. However, spectacles independence can be achieved with monofocal IOLs. With advanced technology for biometry IOL calculations and more precise FSL for cataract surgery, eye surgeons also expect that this independence could become more frequent [4-6].

We evaluated the visual performance of different types of optically designed IOLs in eyes submitted to FSL-assisted cataract surgery. Then, we analyzed whether this technology could offer more precise refractive outcomes related to a more precise capsulotomy and effective lens position, resulting in spectacles independence.

\section{MATERIALS AND METHODS}

We conducted a retrospective study of cases operated at a single center in Brazil using the laser platform LenSx/Alcon FSL (Alcon, Fort Worth, TX) with a soft contact lens patient interface. A discussion of the risks and benefits of the procedure was followed by the provision of informed written consent. The study adhered to the tenets of the Declaration of Helsinki and was approved by the local ethics committee. All procedures were performed at the Eye Clinic of São Paulo between June and August 2014. 
Patients with visually significant cataract were included in this study. The exclusion criteria were a history of glaucoma or retinal detachment, corneal disease, irregular corneal astigmatism, abnormal irises, macular degeneration or retinopathy, neuro-ophthalmic disease, or a history of ocular inflammation.

\section{Preoperative and Postoperative Protocol}

All patients underwent a detailed preoperative assessment, including slit-lamp biomicroscopy, tonometry, and measurement of uncorrected and corrected distance visual acuity and manifest refraction. Cataract investigations included measurement of axial length and biometry (IOLMaster V 7.5, Carl Zeiss Meditech Inc., Jena, Germany); pachymetry, corneal topography, and lens densitometry (Pentacam HR, Oculus, Wetzlar, Germany); and specular microscopy (EM-3000, Tomey, Phoenix, AZ).

All procedures were performed with the LenSx laser under topical anesthesia. The disposable patient interface was docked to the eye, and the individual patient treatment was programmed into the laser. The anterior capsulotomy, nuclear-fragmentation, primary and side-port corneal incisions were then created with the FSL under optical coherence tomography (OCT) image control. The treatment parameters for the LenSx FSL are summarized in Table 1.

After completion of the laser procedure, the patient was transferred to the operating room. The laser-cut corneal incisions were opened; the anterior capsular button was removed with forceps, and then the surgery was completed following the standard phacoemulsification procedure using the Infiniti Vision System (Alcon). The postoperative regimen included 1 drop of $1.0 \%$ moxifloxacin (Vigamox, Alcon), ketorolac tromethamine (Acular, Allergan), and $0.1 \%$ dexamethasone (Maxidex, Alcon) 4 times per day for 2 weeks, then dexamethasone drops twice per day for 1 week and once daily for the last week. Each patient was followed up at days 1, 7 and 30 post-operation.

\section{Refractive Outcomes Analysis}

For comparison, all eyes included in the study with 1 month of postoperative follow-up were divided into 2 groups: Group 1 comprised the cases that required a prescription for eyeglasses, and Group 2 comprised the cases that did not require a prescription.

\section{Statistical Analysis}

Statistical analysis was performed with GraphPad Prism version 6 for Mac OS X (GraphPad Software, San Diego, CA, USA). The Mann-Whitney test was used to compare the incidence of variables across the 2 groups, and the Wilcoxon test was used to analyze significance within the 2 groups. Corrective lens prescriptions among the eyes that received monofocal, monofocal toric, multifocal and multifocal toric IOLs were analyzed by the chi-square test. Corrective lens prescriptions for eyes that received monofocal or multifocal IOLs were compared by the Fisher exact test. The level of significance was set at $\mathrm{P}<0.05$ across all parameters.

\section{RESULTS}

A total of 35 unilateral consecutive cataract surgeries from 35 patients ( 22 females and 13 males) were performed with the LenSx FSL and included in the study. The mean age was 65 years ( \pm 11 standard deviation $(\mathrm{SD})$, ranging from 44 to 82). Among the baseline characteristics, the mean cataract nuclear sclerosis was grading $2( \pm 0.5 \mathrm{SD}$, ranging from 1 to 4), the mean axial length was $23.82 \mathrm{~mm}( \pm 1.30 \mathrm{SD}$, ranging from 21.67 to 26.44 ), the mean anterior chamber depth was $3.30 \mathrm{~mm}$ ( $\pm 0.37 \mathrm{SD}$, ranging from 2.41 to 3.89 ) and the mean preoperative endothelial cell density was $2210( \pm 355$ SD, ranging from 1713 to 2958). The preoperative mean best corrected distance visual acuity (BDVA) in logMAR was 0.4 ( $\pm 0.2 \mathrm{SD}$, ranging from 0.1 to 1.3 ), and the postoperative mean uncorrected distance visual acuity (UDVA) in $\log$ MAR was 0.1 ( \pm 0.1 , ranging from 0.0 to 0.4$)$.

\section{Spectacles Independence Analysis}

Of the 35 eyes, 23 received a monofocal IOL (18 monofocal spherical IOLs: 17 SN60WF and 1 MA60AC; and 5 monofocal toric IOL: 2 SN6AT4, 1 SN6AT5 and 2 SN6AT9) and 12 received a multifocal IOL (4 multifocal IOLs: 4 SN6AD1; and 8 multifocal toric IOL: 6 SND1T2, 1 SND1T3 and 1 SND1T4). After 1 month postoperative, 12 cases required a prescription for eyeglasses (Group 1), and 23 did not require a prescription (Group 2). There were no statistically significant differences between the groups during the preoperative period $(\mathrm{P}=0.4748)$. However, postoperatively, the UDVA was significantly worse in eyes requiring correction $(\mathrm{P}=0.0112)$. Additionally, a significant postoperative improvement in visual acuity was present in both groups $(\mathrm{P}<0.0001$, Group 2; $\mathrm{P}=0.0156$, Group 1) (Table 2).

Table 1. The most commonly used treatment parameters for LenSx femtosecond laser.

\begin{tabular}{|c|c|c|c|c|}
\hline & Primary Incision & Side-port Incision & Anterior Capsulotomy & Nuclear Fragmentation \\
\hline \hline Energy $(\mu \mathrm{J})$ & 5.0 & 5.0 & 5.0 & 8.0 \\
\hline Configuration & 3 plane internal-trapezoid & single-plane external trapezoid & 5.0 -mm diameter & $4.8 \mathrm{~mm}$ diameter of 2 chop/2.3mm of 1 cylinder \\
\hline Spot/Layer & $5.0 / 5.0$ & $5.0 / 5.0$ & $4.0 / 3.0$ & $12.0 / 10.0$ \\
\hline Width $(\mathrm{mm})$ & 2.4 & 1.2 & - & - \\
\hline Delta value & - & - & $325 / 325$ & $500 / 800$ \\
\hline ant/post & & & & \\
\hline
\end{tabular}


Table 2. Spectacles independence analysis.

\begin{tabular}{|c|c|c|c|c|c|}
\hline \multirow{2}{*}{} & \multicolumn{2}{|c|}{ Group 1 $(\mathrm{n}=12)$} & \multicolumn{2}{|c|}{ Group 2 (n=23) } & \multicolumn{2}{|c|}{ (Did Not Require Prescription) } \\
\cline { 2 - 6 } & Median & Interquartile Range & Median & $\begin{array}{c}\text { Interquartile } \\
\text { Range }\end{array}$ & $\begin{array}{c}\text { Between group significance } \\
\text { (Mann-Whitney test) }\end{array}$ \\
\hline \hline Preoperative BCVA (logMAR) & 0.4 & 0.3 to 0.6 & 0.3 & 0.2 to 0.4 & $\mathrm{P}=0.4748$ \\
\hline Postoperative UCVA (logMAR) & 0.2 & 0.1 to 0.3 & 0.0 & 0.0 to 0.1 & $\mathbf{P}=\mathbf{0 . 0 1 1 2}$ \\
\hline $\begin{array}{c}\text { Within group significance } \\
\text { (Wilcoxon test) }\end{array}$ & \multicolumn{3}{|c|}{$\mathbf{P}=\mathbf{0 . 0 1 5 6}$} & $\mathbf{P}<\mathbf{0 . 0 0 1}$ & \\
\hline
\end{tabular}

The preoperative best corrected visual acuity (BCVA) and the postoperative uncorrected visual acuity (UCVA) in logMAR in eyes that did or did not require corrective lenses.

When analyzing the groups by dividing the eyes that received monofocal IOLs from those that received multifocal IOLs, the proportion of eyes that did not require corrective lenses was significantly greater $(\mathrm{P}=0.0268)$ in eyes that received multifocal IOLs $(91.67 \%)$ compared with those that received monofocal IOLs (47.83\%) (Fig. 1). Additionally, $100 \%$ of eyes that received a multifocal toric IOL did not require eyeglasses at one month postoperatively (Table $\mathbf{3}$ ).

\section{DISCUSSION}

The importance of having perfectly sized, symmetrical capsulotomies when performing the final refraction is increasingly recognized $[7,8]$. Additionally, age, preoperative and postoperative correct distance visual acuity (CDVA), ocular comorbidity, gender, and the achieved postoperative refraction are related to satisfaction with vision [9]. Other important factors related to poor patient-reported outcomes after surgery are good preoperative assessments, with a precise biometric IOL calculation and postoperative IOL position, and intra- and postoperative complications are independently associated with worse postoperative UDVA $[10,11]$. We found a significant improvement in visual acuity in both groups after cataract surgery $(\mathrm{P}=0.0156$, Group 1; P $<0.0001$, Group 2).

Although the sample size of this study was small, we did not have any surgical complication with the cases of FSL cataract surgeries to report. As shown previously with a large sample size, the surgical outcomes and safety of FSLassisted cataract surgery to improve significantly with greater surgeon experience, development of modified techniques and improved technology [12].

Currently, good unaided distance visual acuity is a realistic expectation after cataract surgery and IOL implantation [13]. Multifocal IOLs provide patients with good distance and near vision, a high rate of spectacles independence, and a high degree of patient satisfaction [14]. However, preoperative factors, such as female gender, low preoperative visual acuity, glaucoma, precision in reaching the target refraction during second-eye surgery, and biometry prediction errors are related to less successful planned postoperative refraction $[15,16]$. Furthermore, patients who

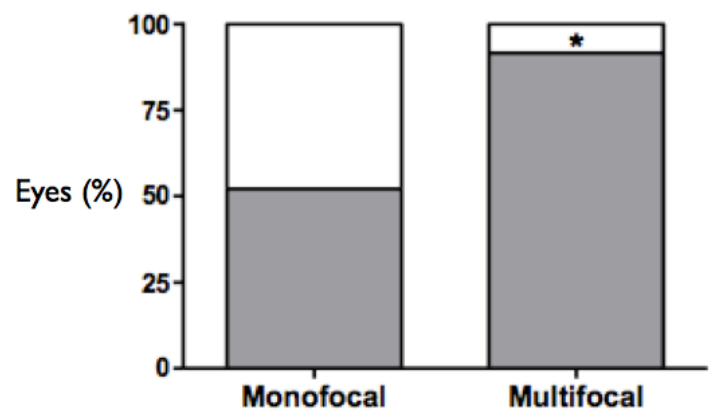

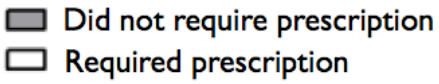

$\square$ Did not require prescription

Fig. (1). Corrective lens prescriptions between eyes that received monofocal $(47.83 \%)$ or multifocal $(8.33 \%)$ IOLs. * P $=0.0268$, Fisher exact test.

Table 3. Corrective lens prescriptions among eyes that received monofocal, monofocal toric, multifocal and multifocal toric IOLs.

\begin{tabular}{|c|c|c|c|}
\hline IOL Types & Group 1 (Required Prescription) & Group 2 (Did Not Require Prescription) & Total \\
\hline \hline Monofocal & $9(50 \%)$ & $9(50 \%)$ & $18(100 \%)$ \\
\hline Monofocal Toric & $2(40 \%)$ & $3(60 \%)$ & $5(100 \%)$ \\
\hline Multifocal & $1(25 \%)$ & $3(75 \%)$ & $4(100 \%)$ \\
\hline Multifocal Toric & $0(0 \%)$ & $8(100 \%)$ & $8(100 \%)$ \\
\hline Total & $12(34 \%)$ & $23(66 \%)$ & $35(100 \%)$ \\
\hline
\end{tabular}


already wear spectacles are expected to wear them after cataract surgery. Those patients who are not already wearing spectacles do not expect to wear them. This latter group is at particular risk for refractive disappointment and complaint [17].

Patients who decide to submit to cataract surgery now have two main options: traditional phacoemulsification and the new technology with FSL. In addition to the financial issues concerning this technology, better and safer outcomes are crucial for making this decision [18]. If the motivation to achieve spectacles independence is the deciding factor, FSLassisted cataract surgery results in a significantly more predictable IOL power calculation than conventional phacoemulsification surgery, most likely is due to a more precise capsulotomy, which results in a more stable IOL position $[19,20]$. In general, patients consider the opportunity to be free of glasses as being very important [17].

When analyzing the refractive outcomes, 23 eyes $(66 \%$ of the cases) were independent of spectacles after the FSL-assisted cataract surgery. They all had a good preoperative assessment, including an IOLMaster biometry, and $100 \%$ of the eyes that had implanted multifocal toric IOLs did not require a prescription.

We evaluated the spectacles independence after cataract surgery with femto laser and different IOLs. We believe one major point is the patient's selection and expectation. Although we realized a fine preoperative evaluation and had precise biometry measurements, had a perfectly sized capsulotomy and uneventful surgeries. We could not evaluate the CDVA and refraction or aberrometric data post operatively, due to the retrospective analysis. Another important point to address as limitation of the present work is the absence of comparison between conventional phacoemulsification and femto surgery which is an ongoing study in our institution. Therefore, more studies are needed to evaluate these outcomes. The significance of these findings needs to be further investigated.

\section{CONCLUSION}

The FSL-mediated surgical precision associated with modern IOL technology may be an important factor related to vision satisfaction after cataract surgery.

\section{CONFLICT OF INTEREST}

The authors confirm that this article content has no conflict of interest.

\section{ACKNOWLEDGEMENTS}

Declared none.

\section{REFERENCES}

[1] Sutton G, Bali SJ, Hodge C. Femtosecond cataract surgery: transitioning to laser cataract. Curr Opin Ophthalmol 2013; 24: 3-8.

[2] Kránitz K, Miháltz K, Sándor GL, Takacs A, Knorz MC, Nagy ZZ. Intraocular lens tilt and decentration measured by Scheimpflug camera following manual or femtosecond laser-created continuous circular capsulotomy. J Refract Surg 2012; 28: 259-63.

[3] Filkorn T, Kovács I, Takács A, Horváth E, Knorz MC, Nagy ZZ. Comparison of IOL power calculation and refractive outcome after laser refractive cataract surgery with a femtosecond laser versus conventional phacoemulsification. J Refract Surg 2012; 28: 540-4.

[4] Hawker MJ, Madge SN, Baddeley PA, Perry SR. Refractive expectations of patients having cataract surgery. J Cataract Refract Surg 2005; 31: 1970-5.

[5] Cuq C, Lafuma A, Jeanbat V, Berdeaux G. A European survey of patient satisfaction with spectacles after cataract surgery and the associated costs in four European countries (France, Germany, Spain, and Italy). Ophthalmic Epidemiol 2008; 15: 234-41.

[6] Zhang F, Sugar A, Jacobsen G, Collins M. Visual function and patient satisfaction: Comparison between bilateral diffractive multifocal intraocular lenses and monovision pseudophakia. J Cataract Refract Surg 2011; 37: 446-53.

[7] Ohmi S. Decentration associated with asymmetric capsular shrinkage and intraocular lens size. J Cataract Refract Surg 1993; 19: 640-43.

[8] Cekic O, Batman C. The relationship between capsulorhexis size and anterior chamber depth relation. Ophthalmic Surg Lasers 1999; 30: 185-90.

[9] Rönbeck M, Lundström M, Kugelberg M. Study of possible predictors associated with self-assessed visual function after cataract surgery. Ophthalmology 2011; 118: 1732-8.

[10] Lundström M, Stenevi U. Analyzing patient-reported outcomes to improve cataract care. Optom Vis Sci 2013; 90: 754-9.

[11] Yan X, Guan C, Mueller A, et al. Outcomes and Projected Impact on Vision Restoration of the China Million Cataract Surgeries Program. Ophthalmic Epidemiol 2013; 20(5): 294-300.

[12] Roberts TV, Lawless M, Bali SJ, et al. Surgical outcomes and safety of femtosecond laser cataract surgery: a prospective study of 1500 consecutive cases. Ophthalmology 2013; 120: 227-33.

[13] Calladine D, Evans JR, Shah S, Leyland M. Multifocal versus monofocal intraocular lenses after cataract extraction. Cochrane Database Syst Rev 2012; 9: CD003169.

[14] Cochener B, Fernández-Vega L, Alfonso JF, Maurel F, Meunier J, Berdeaux G. Spectacle independence and subjective satisfaction of ReSTOR multifocal intraocular lens after cataract or presbyopia surgery in two European countries. Clin Ophthalmol 2010; 4: 81-9.

[15] Kugelberg M, Lundström M. Factors related to the degree of success in achieving target refraction in cataract surgery: Swedish National Cataract Register study. J Cataract Refract Surg 2008; 34: 1935-9.

[16] Behndig A, Montan P, Stenevi U, Kugelberg M, Zetterström C, Lundström M. Aiming for emmetropia after cataract surgery: Swedish National Cataract Register study. J Cataract Refract Surg 2012; 38: 1181-6.

[17] Hawker MJ, Madge SN, Baddeley PA, Perry SR. Refractive expectations of patients having cataract surgery. J Cataract Refract Surg 2005; 31: 1970-5.

[18] Uy HS, Edwards K, Curtis N. Femtosecond phacoemulsification: the business and the medicine. Curr Opin Ophthalmol 2012; 23: 33-9.

[19] Leyland M, Zinicola E. Multifocal versus monofocal intraocular lenses in cataract surgery: a systematic review. Ophthalmology 2003; 110: 1789-98.

[20] Filkorn T, Kovács I, Takács A, Horváth E, Knorz MC, Nagy ZZ. Comparison of IOL power calculation and refractive outcome after laser refractive cataract surgery with a femtosecond laser versus conventional phacoemulsification. J Refract Surg 2012; 28: 540-4. 